\title{
Single Frontal Burr-hole Craniostomy Under Local Anaesthesia for Treating Chronic Subdural Haematoma in the Septuagenarians and Older Adults: Surgical Technique and Results
}

\section{Amos Olufemi Adeleye ( $\square$ femdoy@yahoo.com )}

Division of Neurological Surgery, Department of Surgery, College of Medicine, University of Ibadan, and University College Hospital, UCH, Ibadan, Nigeria

\section{Bartholomew Ulasi}

Department of Surgery, University College Hospital, UCH, Ibadan

\section{Research}

Keywords: chronic subdural haematoma, burr-hole craniostomy, single burr-hole, local anaesthesia, geriatric patients

Posted Date: November 30th, 2020

DOl: https://doi.org/10.21203/rs.3.rs-112015/v1

License: (c) (1) This work is licensed under a Creative Commons Attribution 4.0 International License. Read Full License 


\section{Abstract}

Introduction. Chronic subdural haematoma is not a totally benign disease in the elderly patients, especially those aged 70 years and above. Hence, the surgical treatment of CSDH in those aged patients needs to be as minimally disruptive as possible

Materials and Methods. An annotated description of the surgical technique of single frontal burr-hole craniostomy performed under local anaesthesia and without closed tube drainage for evacuating CSDH in the septuagenarians and older adults is described. We also review the outcome of the procedure on a prospective consecutive cohort of patients in our service

Results. Thirty patients, 25 males, aged 70 years and above successfully underwent this surgical procedure. Their mean age was 76.5 years (standard deviation, SD, 4.3). The CSDH was bilateral in $30 \%$ (9/30); more left-sided in the rest, the unilateral cases, and was of mixed density radiologically, in most cases $(73 \%, 22 / 30)$. Clinical presentation was mainly gait impairment, cognitive decline, and headache; and $57 \%(17 / 30)$ presented in coma or stupor as assessed by the Markwalder grading scale.

The surgery was successfully executed in all, median duration 45.0 minutes (IQR 37.3-60.0), and solely under local anaesthesia in $77 \%(23 / 30)$ or with momentary short-acting sedation in the rest. Outcome was very good in $83.3 \%$ (25/30) using the modified Rankin Scale (mRS) including two-third (20/30) completely asymptomatic (mRS 0 ), and $5 / 30$ with only slight symptoms and no disability, mRS 1 . Three patients died perioperatively.

Conclusion. This surgical technique appears very effectual for CSDH, is executed at a comparatively lowcost, and, being much less disruptive than other traditional methods, may be a more homeostatic operative treatment in geriatric patients at least.

\section{Introduction}

Non-acute subdural haematoma, or chronic subdural haematoma (CSDH), is a neurosurgical presentation that is easily treated by very simple operative paradigm. But it is a condition that is wont to be seen more frequently in the elderly, accounting for up to $58 / 100,00$ of people aged 70 years and above in the West. ${ }^{1}$ The neurosurgical operative treatment of $\mathrm{CSDH}$ ranges from the complex undertaking of major cranial openings with or without membranectomy, through burr-hole craniostomy, to the very simple cranial trephinations, including bedside twist drill craniostomy or even bedside subdural tapping with the Aoki needle. ${ }^{2-7}$

But, partly because of existing comorbidities as well as frailty that are not infrequently associated with these senior citizens, CSDH is not a benign disease in the elderly as such. ${ }^{4,8}$ Therefore, it is of some practical necessity to choose operative techniques that are not only effectual but are also as minimally disruptive as possible in the very aged patient population with this condition. The operative procedure of single frontal burr-hole craniostomy performed under local anaesthesia and without subdural tube 
drainage is the technique we deploy in our service for this condition in the elderly patients aged 70 years and above.

In this report we present this surgical technique as a simple, intuitive, minimally-invasive, even low-cost, operative treatment for non-acute subdural haematoma in this not-infrequently frail geriatric patients population.

\section{Materials And Methods}

We present an annotated description of the surgical technique of single frontal burr-hole craniostomy performed under local anaestheisa, and without subdural drain, for $\mathrm{CSDH}$; followed by a descriptive analysis of its use in a consecutive cohort of patients.

\section{Surgical Technique}

A scalp-incision line of two-finger breadth is planned in the frontal region, usually around the hairline for those so blessed. The operative field is then scrubbed and prepped as usual. Next, scalp infiltration with a local anaesthetic agent is made along the proposed incision line as well as along a concentric circle of scalp of about three-finger breadths to facilitate securing the op-site isolation draping with skin staples, Fig. 1. The scalp incision is developed down to the cranium, and a $20 \mathrm{~mm}$ or so burr-hole is placed. The dura is coagulated, and opened generously for all the diameter of the burr-hole. Some of the subdural fluid, usually a little fraction, would gush out spontaneously following this; and then a tube for evacuating the haematoma, usually a $16 \mathrm{~F}$ nasogastric tubing (NGT) is introduced, and directed posteriorly to the parieto-occipital depth of the subdural fluid cavity. The tube must be introduced with only a 2-finger grip, and must not be forced in, Fig. 1. Next, gentle suction pressure is applied to the NGT with a $10 \mathrm{ml}$ syringe (or the like) until the subdural fluid begins to flow out, gravity aided, by capillary action. The whole haemorrhagic content of the subdural space is thus emptied by sump into a bowl, Fig. 2. Using the operating irrigation bulb or syringe also with a curved 'mouth', a thorough irrigation of the subdural space is then achieved, till clear, by introducing warm normal saline in all directions and allowing the subdural irrigation fluid to drain via the NGT still in-situ, Fig. 2e. This is aided by gravity and occasional application of suction pressure to the external ostium of the NGT. The operative wound is then closed as usual.

The patients usually make such brisk recovery, many times on the operating table, that their in-hospital follow-up is usually only by the clinical course. Because of the severe resource-constraints of our lowresource practice, acquisition of postoperative cranial CT, in-hospital or at out-patient follow-up, is not routine, but dictated by clinical indications.

\section{Clinical Materials}

We performed a descriptive analysis of a consecutive cohort of our elderly patients, aged 70 years and above, who have undergone this operative procedure. This analysis includes the demographics (age, 
gender, and some pre-morbid clinical parameters); clinical presentation; the radiological characteristics of the haematoma; the surgical details, and the postoperative outcome.

\section{Results}

There were 30 cases, 25 males and 5 females, aged 70 years and above, mean 76.5 years (SD, 4.3) for this analysis. Of these 30 patients, 16 cases (53.3\%) were 75 years and above; while 8 cases $(26.7 \%)$ were 80 years and above.

Table 1 shows some of the radiological and clinical characteristics of the study subjects. The non-acute subdural haematoma was bilaterial in $30 \%(9 / 30)$; the rest were unilateral, either right or left, with the left having greater frequency, Fig. 3. Computed tomography (CT) scanning was the main radiologic diagnostic investigation in our patients; only a few got magnetic resonance imaging (MRI) and these were usually those for whom other neurological diagnoses (including brain tumour or stroke) were suspected by the primary-care physicians/neurologists before referral for neurosurgery. Radiologically the haematoma on CT was of mixed density in $73 \%(22 / 30)$, the rest hypo- or isodense. Figure 4 shows one of the cases of bilateral CSDH operated with this surgical technique

Table 1

Clinical and radiological characteristics of the geriatric patients treated with this surgical technique

\begin{tabular}{|ll|}
\hline Clinical-Radiological Characteristics & Number (\%) \\
\hline Laterality of subdural haematoma & $9(30)$ \\
Reft & $12(40)$ \\
Bilateral & $9(30)$ \\
Type of subdural by CT density & $5(16.7)$ \\
Hypodense & $22(73.3)$ \\
Mixed density & $3(10.0)$ \\
Isodense & \\
Presenting Symptoms & $29(96.7)$ \\
Gait impairment & $25(83.3)$ \\
Cognitive decline & $23(76.7)$ \\
Headache & $19(63.3)$ \\
Loss of consciousness & \\
\hline
\end{tabular}


Preceding history of trauma was obtained in 18/30 (60\%). Most presented with gait impairment, cognitive decline, and headache, Table 1; and as shown in Table 2, as many as $17 / 30(56.7 \%)$ presented in stupor or coma as assessed with the Markwalder grading scale. ${ }^{9-11}$ Some $40 \%(12 / 30)$ had background history of hypertension and 3 were on low-dose $(75 \mathrm{mg})$ aspirin treatment; $10(33.3 \%)$ had their initial clinical diagnosis as cerebrovascular accident. History of alcohol ingestion was obtained in only 2/30; none had coagulation disorder.

Table 2

The clinical status of the patients, using the Markwalder grading scale, preoperatively and at inhospital discharge

\begin{tabular}{|lll|}
\hline Markwalder Grade & Preoperative & Discharge \\
\hline 0, no symptoms & - & 23 \\
1, mild symptoms & 2 & 4 \\
2, focal deficit & 11 & - \\
3, Stupor & 9 & - \\
4, Coma & 8 & - \\
\hline
\end{tabular}

The surgery was successfully completed in all, median duration 45.0 minutes (IQR 37.3-60.0). It was solely under local anaesthesia in $77 \%$ (23/30); momentary sedation with bolus doses of Propofol, a shortacting agent, was added in the rest for occasional intraoperative restlessness. There was not any episode of intraoperative adverse events. The postoperative recovery was brisk in most patients and the median in-hospital stay before discharge was 7 days (IQR 5-11).

Outcome was very good in $83.3 \%$ (25/30) using the modified Rankin Scale (mRS) including two-third $(20 / 30)$ completely asymptomatic (mRS 0$)$, and $5 / 30$ with only slight symptoms and no disability, (mRS 1). Of the other 5 patients; 2 were $\mathrm{mRS} 2$, with slight disability at discharge. Three patients died, all from those that presented in coma, Table 2. Postoperative CT scanning was acquired in only 8 usually for some concerning postoperative clinical issues. All the patients that were discharged home were followed up in the outpatient department, median duration of $3.5 \mathrm{mo}$ (IQR 2-12); some $70 \%$ of the total patient cohort $(21 / 30)$ have been followed up for more than 3 months.

There were 3 cases of subdural haematoma recurrence, all ipsilateral and in unilateral cases, in our study subjects.

One was actually a failure of the surgery, technically. It involved an 87-year-old man who presented in coma, and was operated for a left-sided mixed-density CSDH with this technique. He made brisk recovery postoperatively; wound staples were removed on POD 6, and was being reviewed for possible in-hospital discharge after which his neurological status progressively declined again. Repeat brain CT on POD 13 revealed a persistent parietal hypodense collection, Fig. 5. In retrospect, this was merely the parietal 
loculation of the initial layered fronto-parietal collection which apparently was ensconced from the frontal subdural component by another capsule, Fig. 5a. At repeat surgery through the same left frontal burr-hole, the outer capsule of this ensconced parietal CSDH was merely sought, and fenestrated generously; the content was emptied, and the cavity irrigated till clear as usual. He thereafter made brisk recovery and was discharged home in excellent clinical state (Markwalder 0, mRS 0), on POD 5. He remains well some 6 years after that surgery. This was early in this surgical experience. We have since learnt to always seek out the inner outer membranes of compartmentalized CSDHs, fenestrate them, and then irrigate them till clear in the subsequent years.

The next recurrence involved an 82-year-old woman who made good recovery, mRS grade 0 , from the first surgery (Fig. 6a, b), but 2 months later showed clinical and radiological recurrence, Fig. 6c). She was reoperated through the same frontal burr-hole; had good recovery, Fig. 6d, and in-hospital outcome, again, mRS 0; and was followed-up for 8 months in clinic before discharge.

The last case of recurrence in this series involved a 76-year-old man who recovered briskly from the first surgery, and was discharged home mRS 0, on POD 4; was seen as outpatient on POD 10 for stitch removal, but then represented on POD 16 with clinical and radiological recurrence. He was re-operated with good recovery, mRS 1 , too.

\section{Discussion}

The operative treatment recommended for $\mathrm{CSDH}$ in standard neurosurgical texts ranges from the extreme to the very simple. The former includes especially 'aggressive large craniectomy and stripping of neomembranes' ${ }^{\prime 2,13}$; and the latter, much less invasive procedures like burr-hole craniostomy, twist drill craniostomy ${ }^{14,15}$ with or without the use of the Subdural Evacuating Port System (SEPS), ${ }^{16}$ or percutaneous subdural tapping using the Aoki needle. ${ }^{2,17}$ Out of all these surgical techniques, burr-hole craniostomy, usually double but occasionally single, appears to be the most common technique among neurosurgeons worldwide. Traditionally, this involves placement of a 10-20 mm trephination in the frontal and parietal skull ipsilateral to the haematoma. It is performed usually in the operative room under general anaesthesia. ${ }^{4,18}$

Each of the above surgical techniques has its proponents, and this is one of the main reasons for the perennial controversy surrounding the neurosurgical management of $\mathrm{CSDH}$. But whatever the surgical procedure preferred by each operator, the goals for the operative treatment of non-acute subdural haematoma are fairly straight forward: decompression of the brain parenchyma via the removal of the space-occupying subdural haemorrhagic collection, as well as dilution, and inactivation of the endogenous fibrinolytic agents that osmotically serve to propagate the space-occupying increment in the volume of the residual subdural fluid. ${ }^{1,12}$

The surgical technique presented in this report achieves this purpose, combining simplicity of execution with low cost, and an especially more homeostatic surgical and anaesthetic exertion on the not 
infrequently frail patients, the very elderly population for whom it was devised in our practice. It also so affords opportunity for generous irrigation, till clear, of the subdural space we do not place a subdural drain post haematoma evacuation, nor have need for any unnecessary postoperative CT imaging to follow the resolution of the haematoma. ${ }^{19} \mathrm{~A}$ single burr-hole placed under under local anaesthesia is so close to being so minimally-invasive that most of our patients make brisk recovery right on the operation table. The frontal location is chosen so as to reduce to the barest minimum the amount of intracranial air following generous subdural irrigation during the procedure, Fig. $6 \mathrm{~d}$. This helps preempt the possible complication of tension pneumocephalus.

There are several previous reports of single burr-hole craniostomy for the operative treatment of $\mathrm{CSDH}^{12,20-24}$ But some of them are either designed to be performed under general anaesthesia; ${ }^{4,25}$ or, are usually placed at the epicenter of the intracranial collection, somewhat posteriorly about the parietal region, that the risks of post-procedure intracranial pneumocoeles are higher; ${ }^{12}$ or do not include the means for total evacuation of the subdural haematoma, nor for the generous irrigation of the subdural cavity like our own. Most such procedures thus deploy subdural drains, 2,10,18,24 and are usually CTintensive for postoperative monitoring for recurrence. ${ }^{12,24}$

With the same goal of operative simplicity of single burr-hole for $\mathrm{CSDH}$, the procedure described by Benzel EC et al. ${ }^{12}$ comes close to ours in similarity. It is a single burr-hole, also augmented with a redrubber tube cannulation of the subdural space for evacuating the haematoma, and multi-quadrant subdural space irrigation. They also do not use postop subdural drain. However, their own burr-hole is not exactly as frontal as ours. Hence its needing some neck manipulation to prevent pneumocephalus, (which was impossible in one of their patients with cervical spondylosis, and who was thus complicated with symptomatic pneumocephaulus). They also performed their own procedure under general anaesthesia. We have recently indicated that general anaesthesia costs up to 10 times that of local anaesthesia for neurosurgical operative procedures in our practice. ${ }^{26}$

\section{Pearls and Pitfalls}

Most of our patients tolerate this surgical procedure well. An occasional patient may have moments of restlessness and or blood pressure elevation. These are usually mitigated with the administration of appropriate doses of the short-acting sedative, Propofol. There is also the occasional patient that does not hold the head still in the surgical position during the scalp infiltration of the local anaesthetic, before the full operative field draping. For these, a yet-to-be-scrubbed surgical assistant can help hold the patient's head in position till the injection of the local is completed. Such patients are usually calm thereafter.

One of the main features of this surgical technique is the introduction of the NG tube into the subdural space to help ensure near-complete evacuation of the usually dirty ('engine oil') subdural space contents in CSDH. It is intuitively in this step that the main pitfall to keep in view with this surgical technique inhere. This is the possibility of brain penetration/laceration during the introduction of the NG tube into 
the subdural space. For, in most cases of CSDH the subdural collection creates enough room, extraaxial, for the safe introduction of the tube in the first instance; and the brain re-expansion is usually slow enough to permit multiple exits and re-introduction of the NG tube into different quadrants of the subdural space as deemed appropriate for a more complete brain washing. But in the occasional case where brain re-expansion is brisk after the evacuation of the subdural fluid following the initial introduction of the NG tube into the subdural space, one must need restrain from further attempt(s) at cannulating the subdural space to avoid this pitfall of brain penetration/laceration.

\section{Limitations}

In all, this report is still limited by the fact of it being a single-surgeon report. Also, the number of the study subjects could certainly be larger than what is. However, the smallness of the series actually merely reflects the highly selected nature of the patients, the septuagenarians and older adults, for whom we first used the technique as reported here. We have since found out that most, if not all, patients (young or old; strong or frail) can actually safely undergo this surgical procedure for $\mathrm{CSDH}$. It is, matter of fact, now our de-facto technique for this surgery, and a subject for a prospective cohort study.

\section{Conclusion}

The technique of single frontal burr-hole craniostomy under local anaesthesia, and without post-operative tube drainage presented in this report is an effectual, less-invasive, and low-cost treatment for chronic subdural haematoma; and may be a more homeostatic technique to adopt in the usually frail geriatric patients in particular. This technique thus offers a much simpler way to treat a condition, chronic subdural haematoma, that is very common in neurosurgical practice. It is also much less expensive, making it relevant in areas of the world with low resources

\section{Abbreviations}

CSDH

chronic subdural haematoma; $\mathrm{CT}$ = computed tomography; IQR = interquartile range; $\mathrm{MRI}=$ magnetic resonance imaging; $\mathrm{mRS}=$ modified Rankin scale; $\mathrm{NGT}=$ nasogastric tube; $\mathrm{POD}=$ postoperative day; $\mathrm{SD}=$ standard deviation

\section{Declarations}

Ethical approval and consent to participate: A written consent had been obtained from the Directorate of Clinical services, research and training of the author's university teaching hospital for the audit of his operative surgical record. All the patients gave written, informed consent for the surgical procedure

Consent for publication: A written, informed consent was obtained from each patient or their relations where appropriate for the images used in this report 
Availability of data materials: The clinical data used for this study are available in paper forms and electronic spreadsheet on request, if needed. Please contact the corresponding author for data request

Competing Interest: The authors declare that they have no competing interests

Funding: There was no external funding of any sort received towards this project

\section{Authors' contribution}

AOA: Conceptualization, Methodology/Surgical Technique evolution, Manuscript drafts through all the stage, Project supervision, Approval of submitted version. BIU: Data curation, Analysis, Manuscript drafts, Approval of submitted version. All authors read and approved the final manuscript

Aknowledgement: Not applicable

Authors' information: Not applicable

\section{References}

1. Liu W, Bakker NA, Groen RJ. Chronic subdural hematoma: a systematic review and meta-analysis of surgical procedures. J Neurosurg 2014;121:665-73.

2. Aoki N. Percutaneous subdural tapping for the treatment of chronic subdural haematoma in adults. Neurol Res 1987;9:19-23.

3. Camel M, Grubb RL, Jr. Treatment of chronic subdural hematoma by twist-drill craniotomy with continuous catheter drainage. J Neurosurg 1986;65:183-7.

4. Miranda LB, Braxton E, Hobbs J, Quigley MR. Chronic subdural hematoma in the elderly: not a benign disease. J Neurosurg 2011;114:72-6.

5. Mobbs R, Khong P. Endoscopic-assisted evacuation of subdural collections. J Clin Neurosci 2009;16:701-4.

6. Weigel R, Schmiedek P, Krauss JK. Outcome of contemporary surgery for chronic subdural haematoma: evidence based review. J Neurol Neurosurg Psychiatry 2003;74:937-43.

7. Williams GR, Baskaya MK, Menendez J, Polin R, Willis B, Nanda A. Burr-hole versus twist-drill drainage for the evacuation of chronic subdural haematoma: a comparison of clinical results. $\mathrm{J}$ Clin Neurosci 2001;8:551-4.

8. Borger V, Vatter H, Oszvald A, Marquardt G, Seifert V, Guresir E. Chronic subdural haematoma in elderly patients: a retrospective analysis of 322 patients between the ages of 65-94 years. Acta Neurochir (Wien) 2012;154:1549-54.

9. Markwalder TM. Chronic subdural hematomas: a review. J Neurosurg 1981;54:637-45.

10. Markwalder TM. The course of chronic subdural hematomas after burr-hole craniostomy with and without closed-system drainage. Neurosurg Clin N Am 2000;11:541-6. 
11. Markwalder TM, Steinsiepe KF, Rohner M, Reichenbach W, Markwalder $\mathrm{H}$. The course of chronic subdural hematomas after burr-hole craniostomy and closed-system drainage. J Neurosurg 1981;55:390-6.

12. Benzel EC, Bridges RM, Jr., Hadden TA, Orrison WW. The single burr hole technique for the evacuation of non-acute subdural hematomas. J Trauma 1994;36:190-4.

13. Hamilton MG, Frizzell JB, Tranmer BI. Chronic subdural hematoma: the role for craniotomy reevaluated. Neurosurgery 1993;33:67-72.

14. Ramnarayan R, Arulmurugan B, Wilson PM, Nayar R. Twist drill craniostomy with closed drainage for chronic subdural haematoma in the elderly: an effective method. Clin Neurol Neurosurg 2008;110:774-8.

15. Escosa Bae M, Wessling H, Salca HC, de Las Heras Echeverria P. Use of twist-drill craniostomy with drain in evacuation of chronic subdural hematomas: independent predictors of recurrence. Acta Neurochir (Wien) 2011;153:1097-103.

16. Kenning TJ, Dalfino JC, German JW, Drazin D, Adamo MA. Analysis of the subdural evacuating port system for the treatment of subacute and chronic subdural hematomas. J Neurosurg 2010;113:1004-10.

17. Aoki N. Subdural tapping and irrigation for the treatment of chronic subdural hematoma in adults. Acta Neurochir (Wien) 1986;83:75-6.

18. Lee JY, Ebel H, Ernestus RI, Klug N. Various surgical treatments of chronic subdural hematoma and outcome in 172 patients: is membranectomy necessary? Surg Neurol 2004;61:523-7; discussion 7-8.

19. Garrett MC, Bilgin-Freiert A, Bartels C, Everson R, Afsarmanesh N, Pouratian N. An evidence-based approach to the efficient use of computed tomography imaging in the neurosurgical patient. Neurosurgery 2013;73:209-15; discussion 15-6.

20. Han HJ, Park CW, Kim EY, Yoo CJ, Kim YB, Kim WK. One vs. Two Burr Hole Craniostomy in Surgical Treatment of Chronic Subdural Hematoma. J Korean Neurosurg Soc 2009;46:87-92.

21. Kansal R, Nadkarni T, Goel A. Single versus double burr hole drainage of chronic subdural hematomas. A study of 267 cases. J Clin Neurosci 2010;17:428-9.

22. Khadka NK, Sharma GR, Roka YB, et al. Single burr hole drainage for chronic subdural haematoma. Nepal Med Coll J 2008;10:254-7.

23. Stanisic M, Lund-Johansen M, Mahesparan R. Treatment of chronic subdural hematoma by burr-hole craniostomy in adults: influence of some factors on postoperative recurrence. Acta Neurochir (Wien) 2005;147:1249-56; discussion 56-7.

24. Gazzeri R, Galarza M, Neroni M, Canova A, Refice GM, Esposito S. Continuous subgaleal suction drainage for the treatment of chronic subdural haematoma. Acta Neurochir (Wien) 2007;149:487-93; discussion 93.

25. Mondorf Y, Abu-Owaimer M, Gaab MR, Oertel JM. Chronic subdural hematoma-craniotomy versus burr hole trepanation. Br J Neurosurg 2009;23:612-6. 
26. Adeleye AO, Idowu OK, Ghadirpour R, laccarino C. Minicraniotomy under local anaesthesia and monitored sedation for the operative treatment of uncomplicated traumatic acute extradural haematoma. World Neurosurg 2020.

\section{Figures}
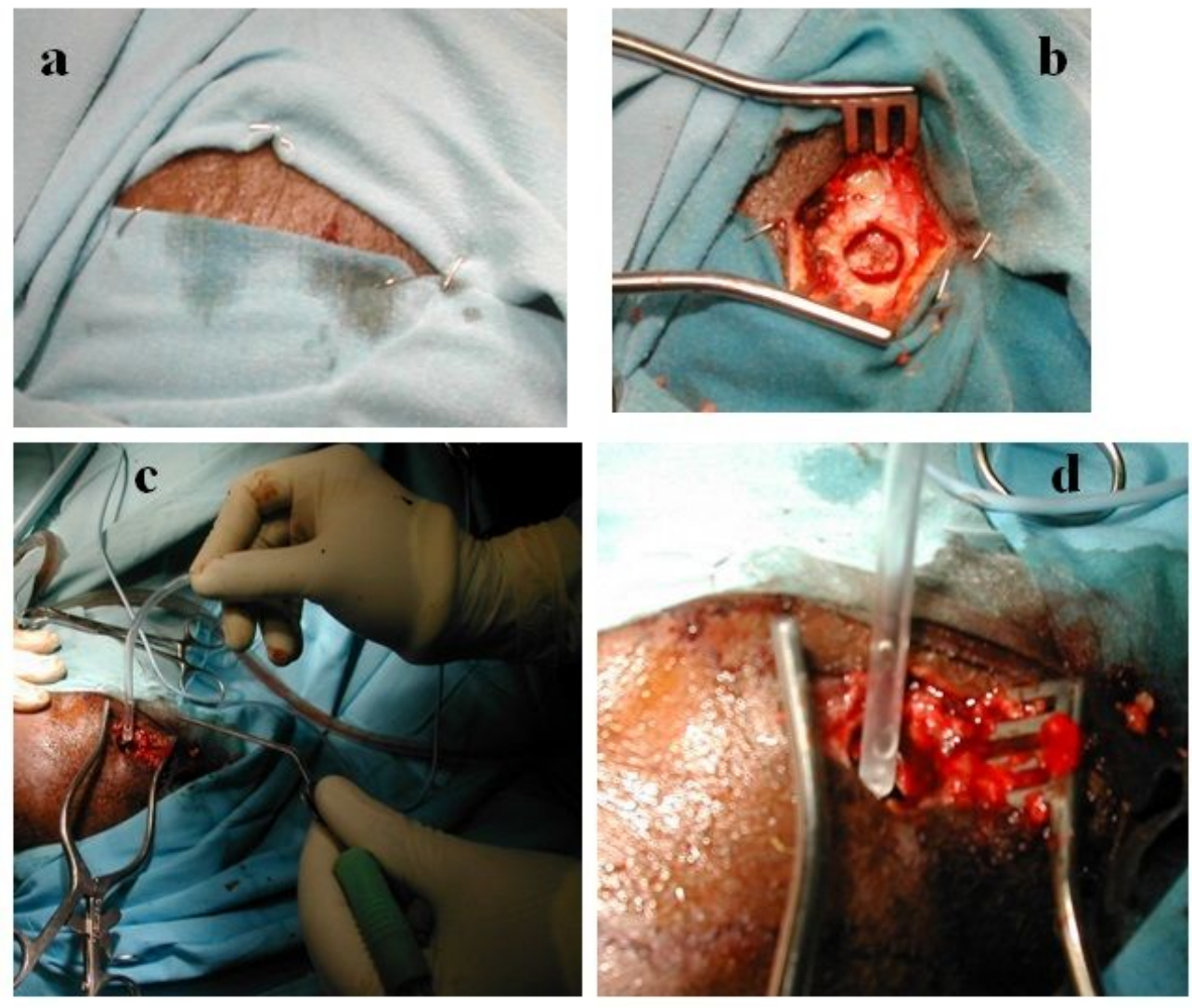

\section{Figure 1}

Single anterior-frontal burr hole evacuation of $\operatorname{CSDH}(\mathrm{a})$ the operative site is chosen about the hair-line, prepped and draped, and local anaesthetic applied (b) a 2-3cm burr hole is placed (c, d) following a generous durotomy involving the full extent of the burr hole a moderate-size NG tube, usually size $16 \mathrm{~F}$, is gently, 2-finger-only-technique, introduced under direct vision into the subdural space and advanced down to its depth 

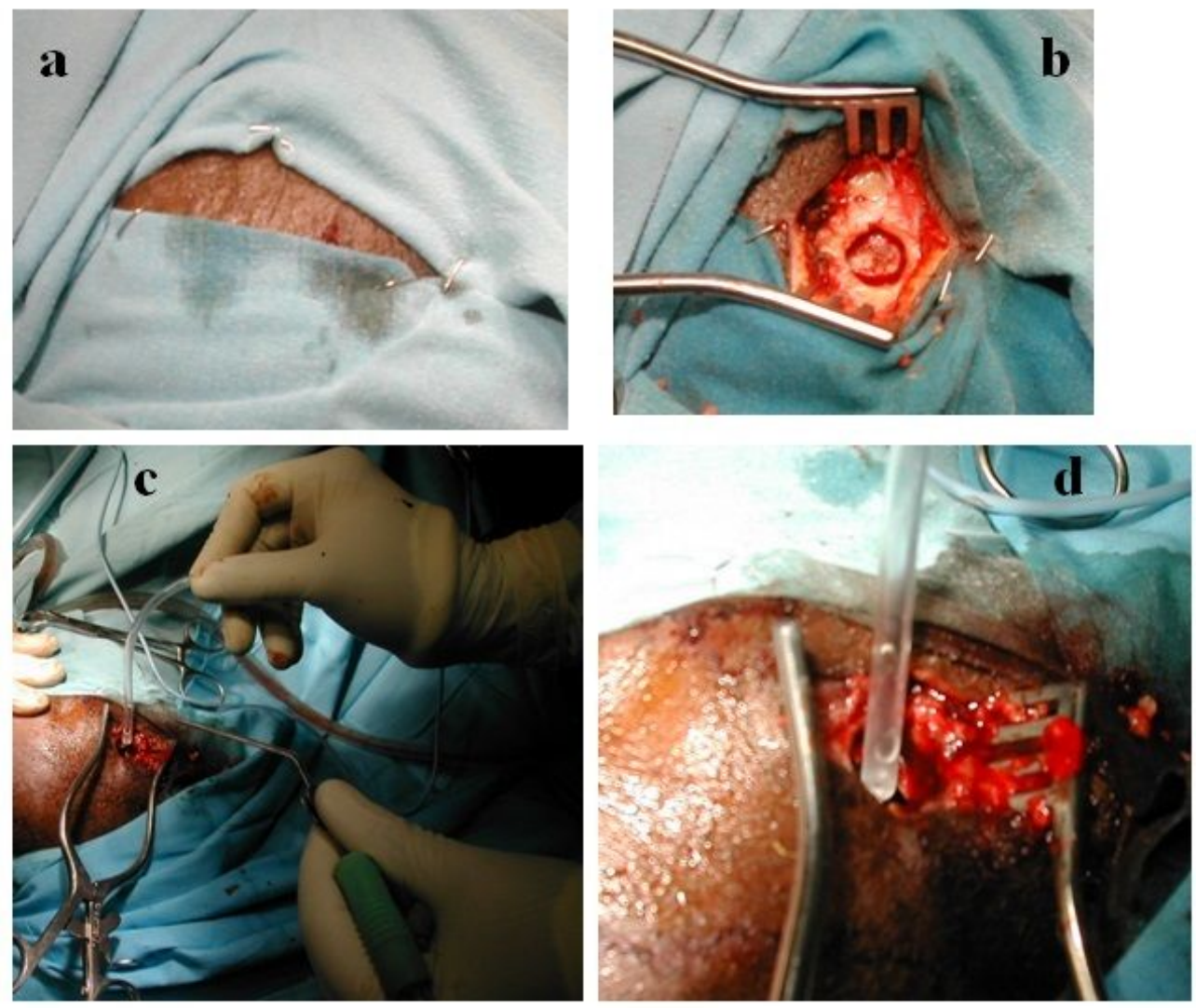

\section{Figure 1}

Single anterior-frontal burr hole evacuation of CSDH (a) the operative site is chosen about the hair-line, prepped and draped, and local anaesthetic applied (b) a 2-3cm burr hole is placed (c, d) following a generous durotomy involving the full extent of the burr hole a moderate-size NG tube, usually size $16 \mathrm{~F}$, is gently, 2-finger-only-technique, introduced under direct vision into the subdural space and advanced down to its depth 

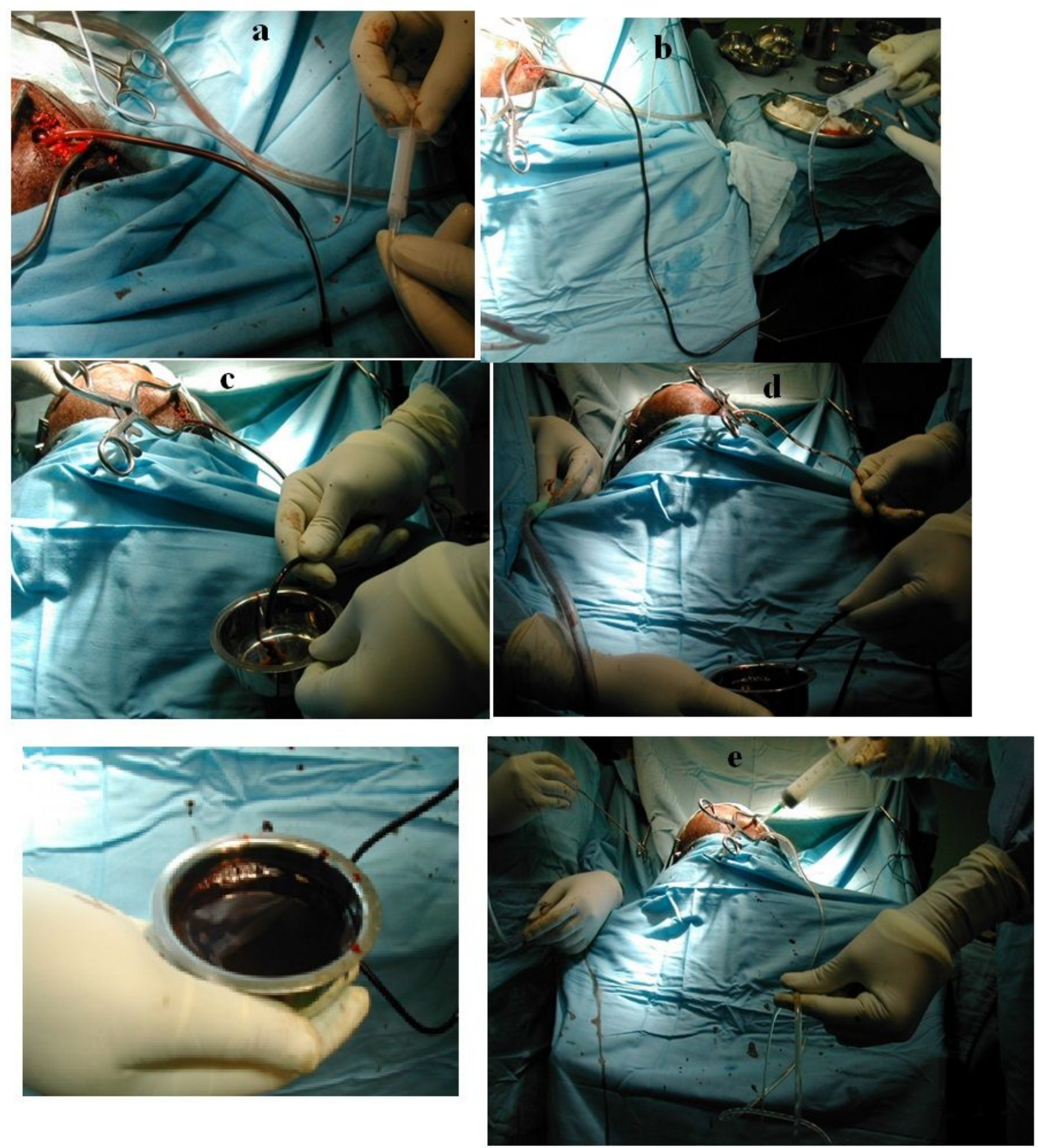

\section{Figure 2}

$(a, b)$ Gentle syringe-suction pressure is applied on the external end of the NG tube until enough blood column is reached in the tube to promote capillary flow of the blood (c) the syringe is withdrawn and the blood flows out spontaneously by capillary action aided by gravity (d) all of the subdural fluid is emptied this way in a controlled manner into a receiver (inset) (e) warm irrigation fluid is introduced via the same burr hole by the side of the NG tube into the 4 quadrants of the subdural space. The fluid also empties 
spontaneously till clear (arrow) by capillarity and gravity via the NG tube. The evacuation tube is then removed and the wound closed in the usual way.
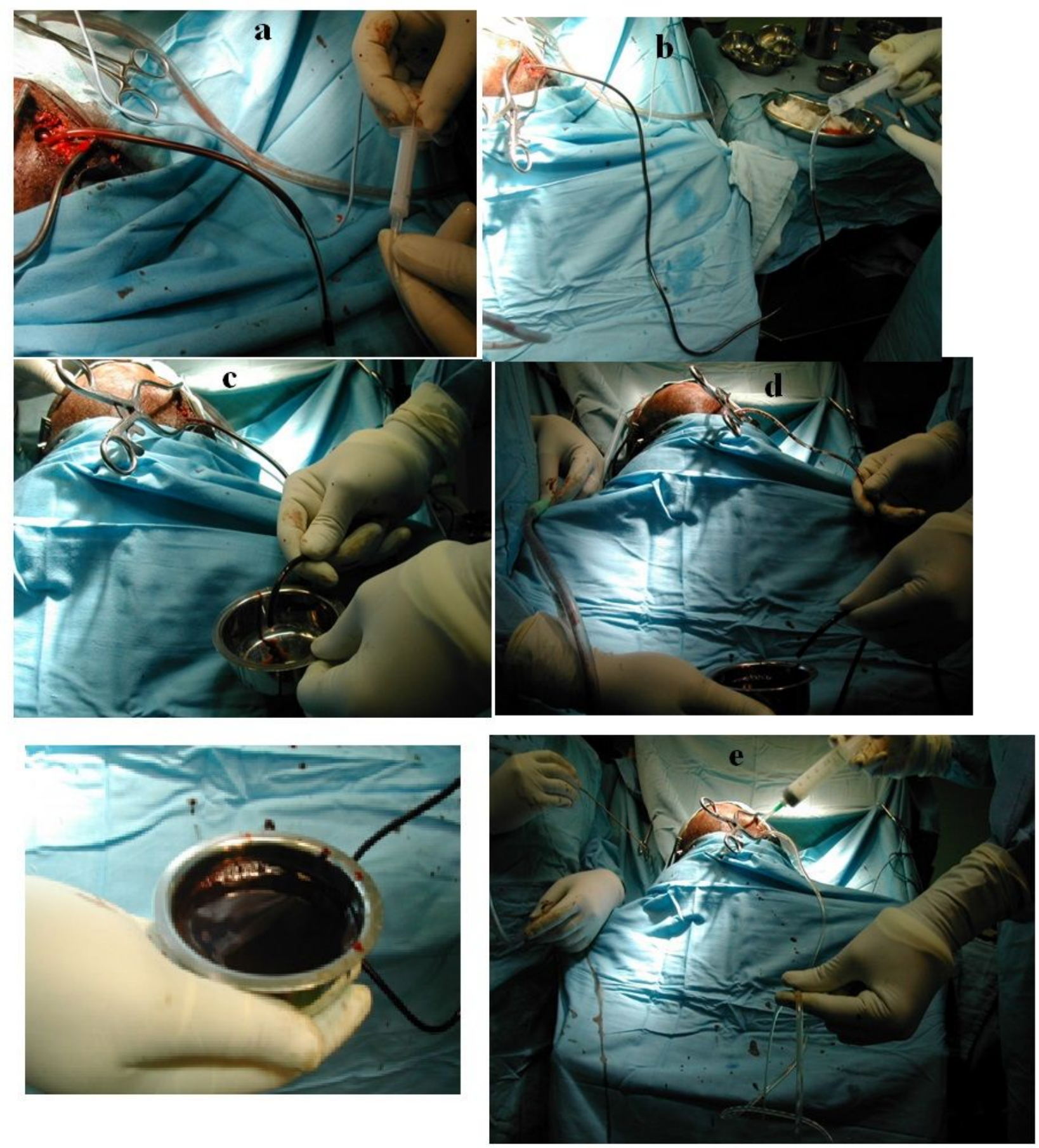

Figure 2

$(a, b)$ Gentle syringe-suction pressure is applied on the external end of the NG tube until enough blood column is reached in the tube to promote capillary flow of the blood (c) the syringe is withdrawn and the blood flows out spontaneously by capillary action aided by gravity (d) all of the subdural fluid is emptied 
this way in a controlled manner into a receiver (inset) (e) warm irrigation fluid is introduced via the same burr hole by the side of the NG tube into the 4 quadrants of the subdural space. The fluid also empties spontaneously till clear (arrow) by capillarity and gravity via the NG tube. The evacuation tube is then removed and the wound closed in the usual way.

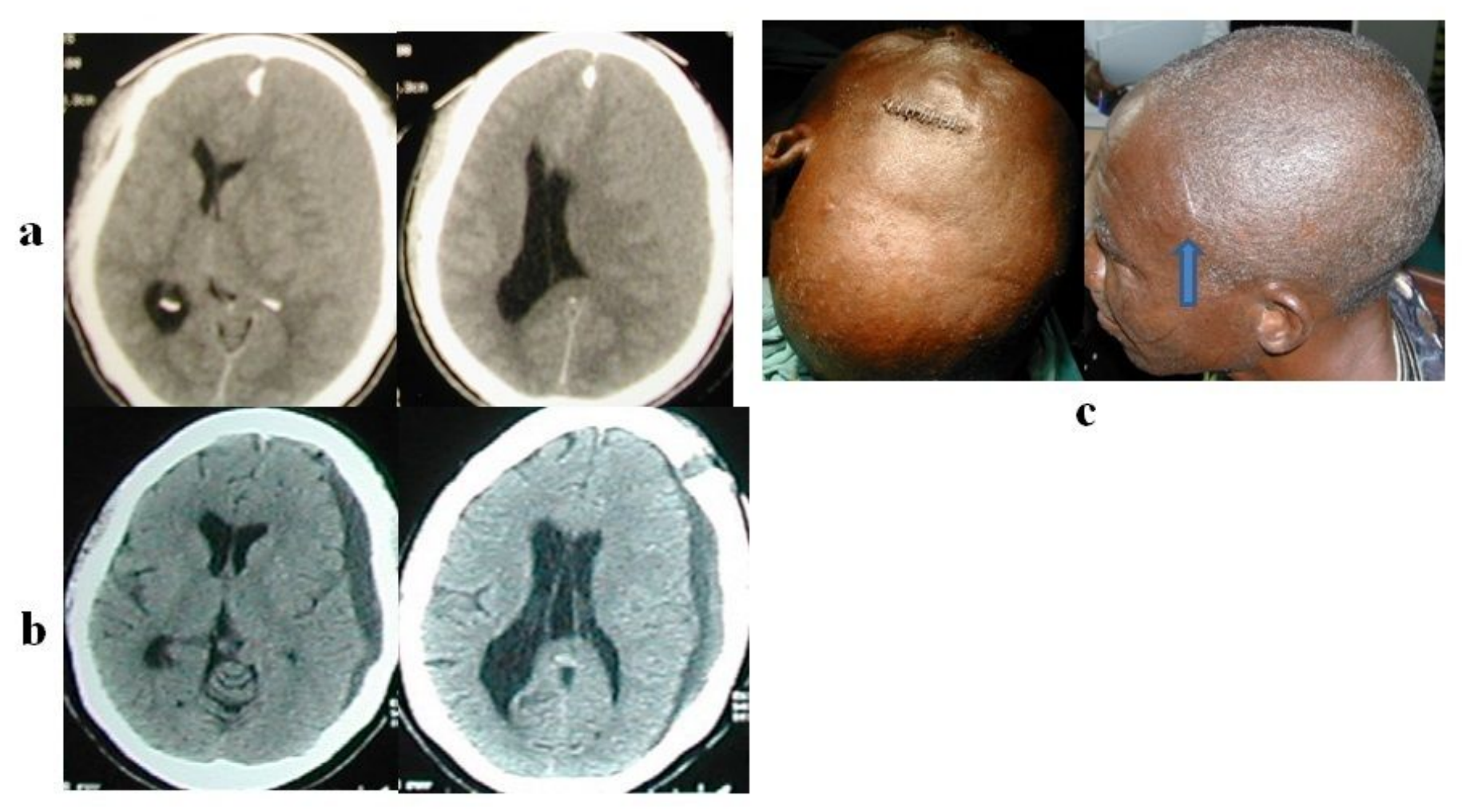

\section{Figure 3}

An elderly man presented unconscious and had cranial CT scan (a) showing left sided subacute subdural haematoma with marked mass effect; (b) cranial CT 5 days post-surgical evacuation via the left anteriorfrontal single burr hole showing marked early postoperative resolution of the intracranial hypertension. The residual subdural collection is a well-diluted hygroma essentially. There is no pneumocephalus (c) surgical incision immediately post op (left figure) and on the first clinic visit 4 weeks post op (arrow, right figure) 


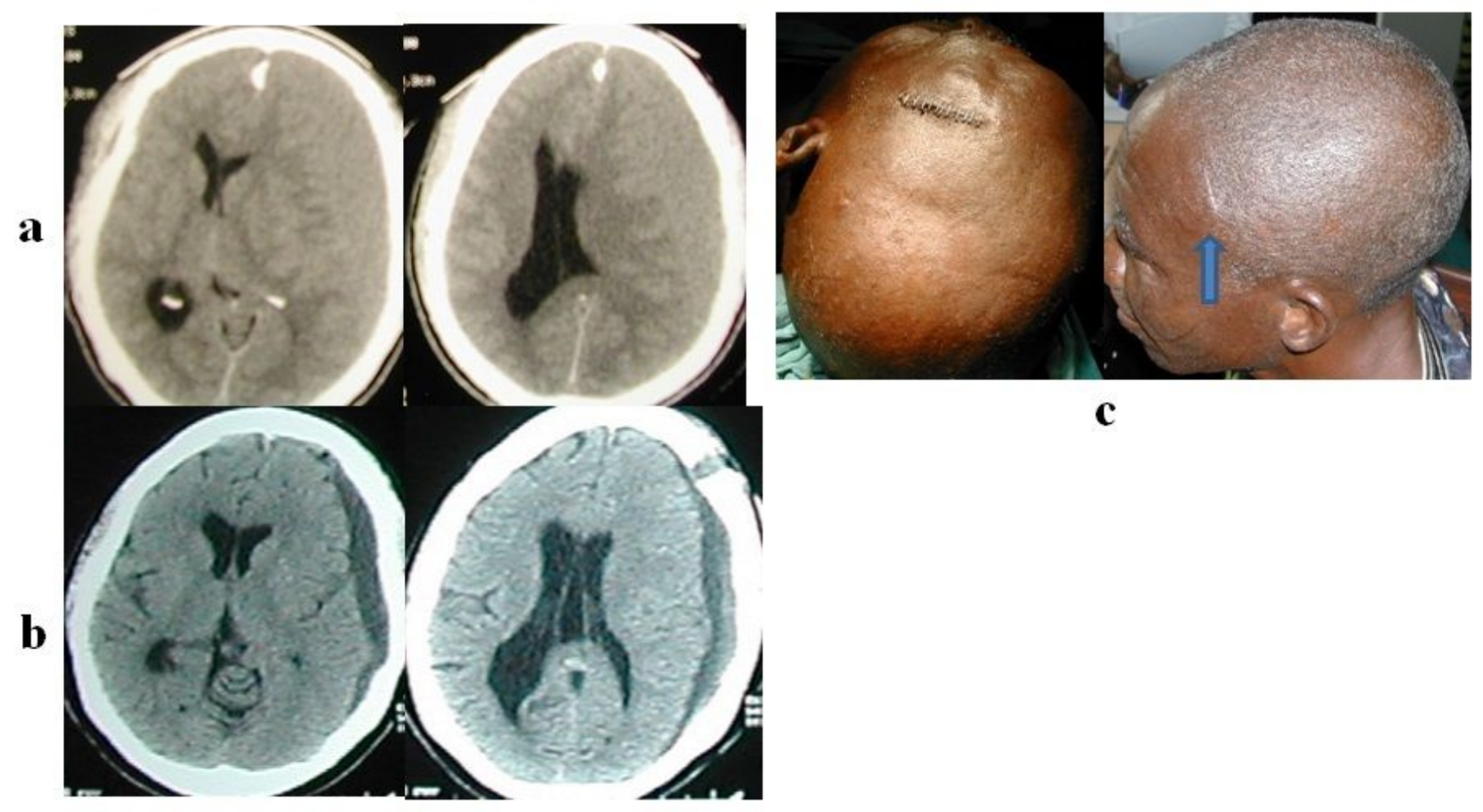

\section{Figure 3}

An elderly man presented unconscious and had cranial CT scan (a) showing left sided subacute subdural haematoma with marked mass effect; (b) cranial CT 5 days post-surgical evacuation via the left anteriorfrontal single burr hole showing marked early postoperative resolution of the intracranial hypertension. The residual subdural collection is a well-diluted hygroma essentially. There is no pneumocephalus (c) surgical incision immediately post op (left figure) and on the first clinic visit 4 weeks post op (arrow, right figure) 

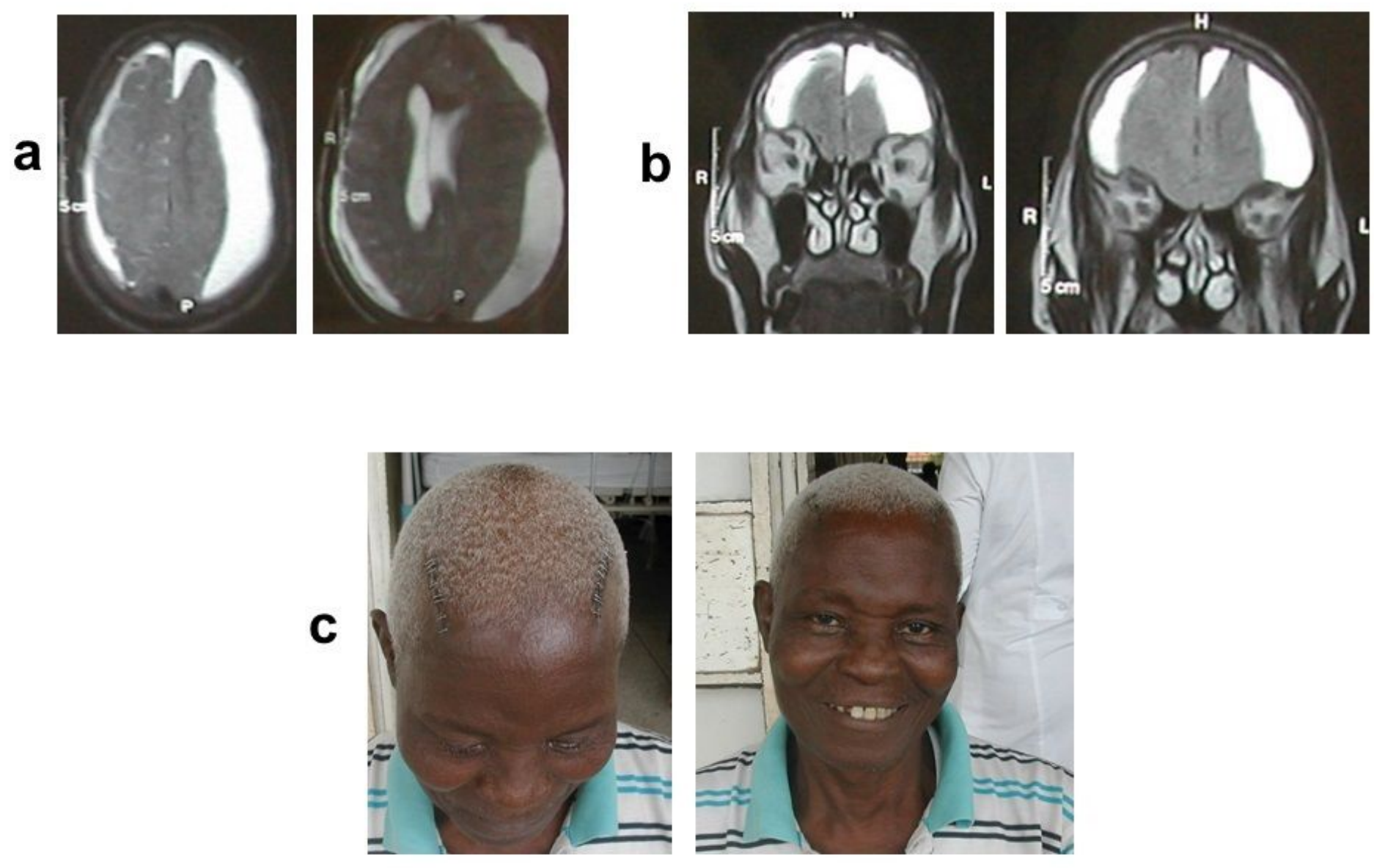

Figure 4

A 78-year-old man was operated with this technique for symptomatic bilateral chronic subdural haematoma. The preoperative MRI cuts are shown in the (a) axial and (b) coronal cross sections (c) The patient, on the postoperative day 7 when the wound staples were removed. 

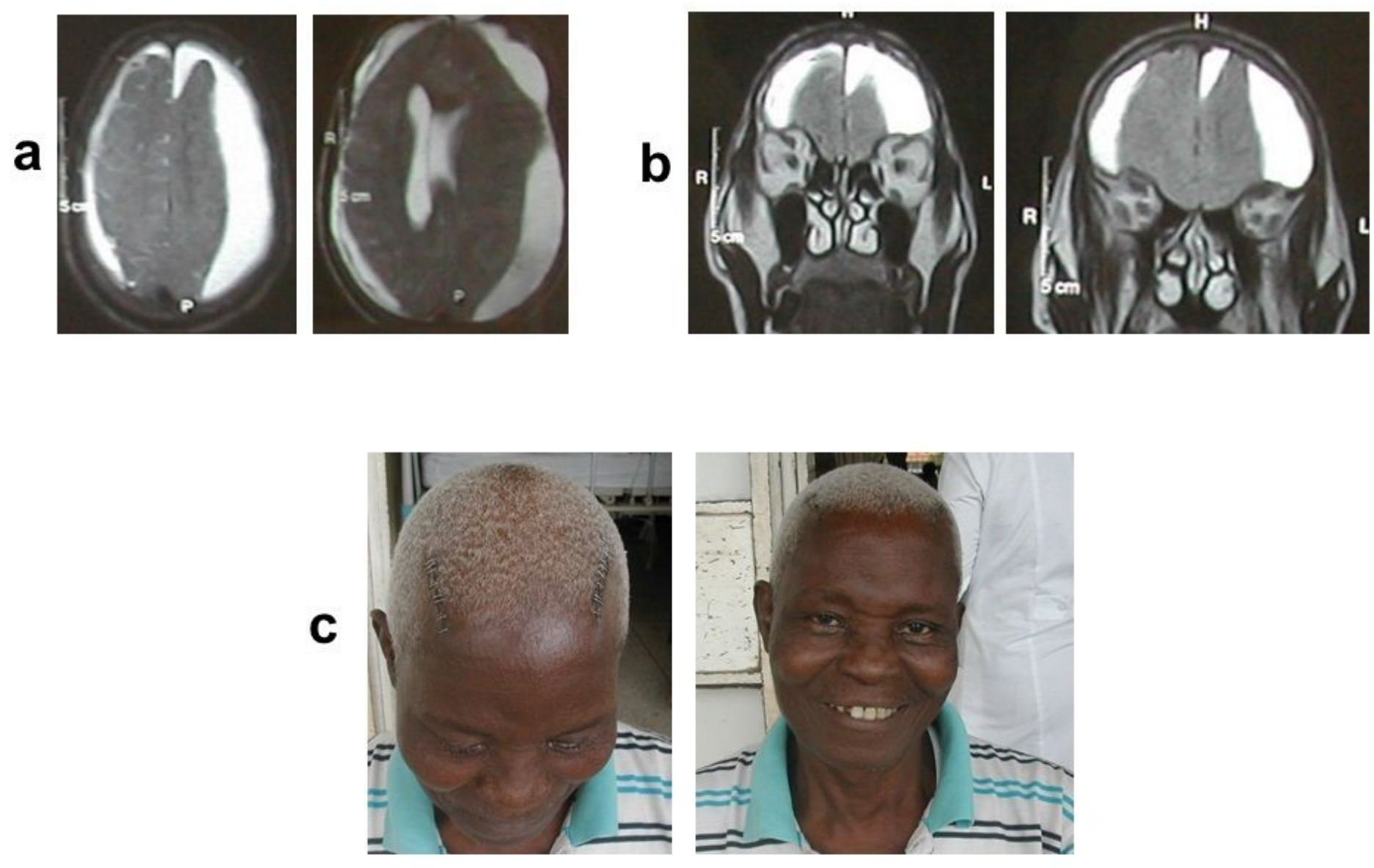

Figure 4

A 78-year-old man was operated with this technique for symptomatic bilateral chronic subdural haematoma. The preoperative MRI cuts are shown in the (a) axial and (b) coronal cross sections (c) The patient, on the postoperative day 7 when the wound staples were removed. 

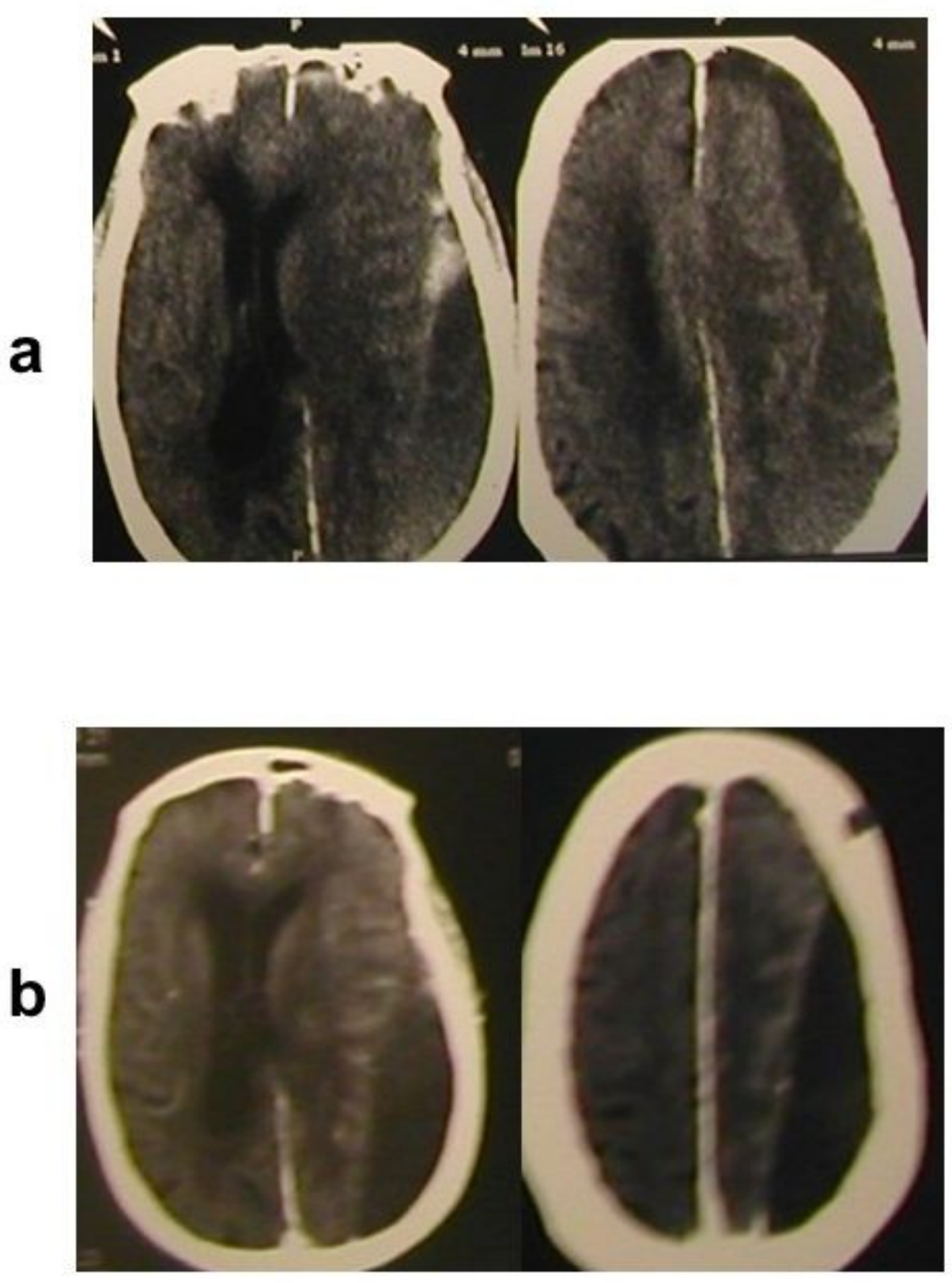

\section{Figure 5}

An 87-year-old man was operated for (a) a left-sided layered CSDH of mixed density. He made initial brisk clinical recovery, then relapsed. Repeat cranial CT on POD 13 showed resolution of the frontal component of the initial lesion, but a persistence of the parieto-occipital component, figure $5 b$. 

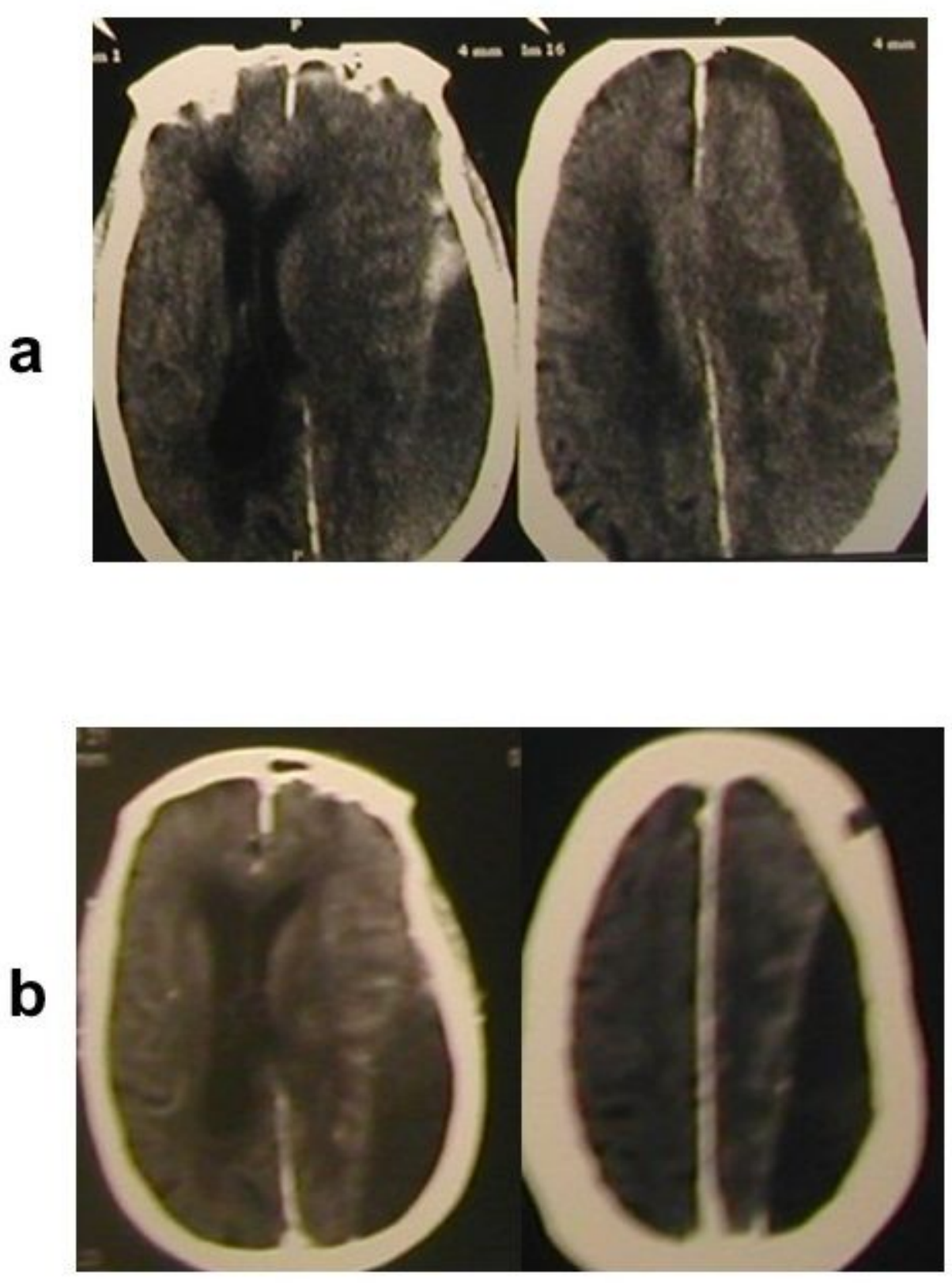

\section{Figure 5}

An 87-year-old man was operated for (a) a left-sided layered CSDH of mixed density. He made initial brisk clinical recovery, then relapsed. Repeat cranial CT on POD 13 showed resolution of the frontal component of the initial lesion, but a persistence of the parieto-occipital component, figure $5 b$. 
a

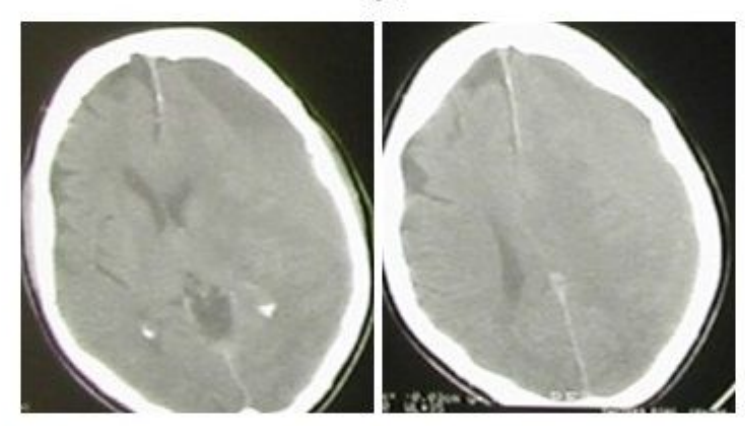

C

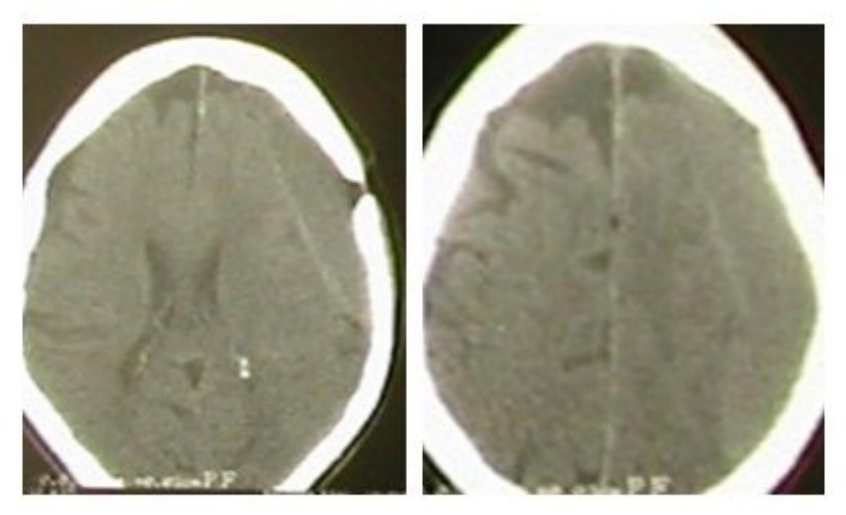

b

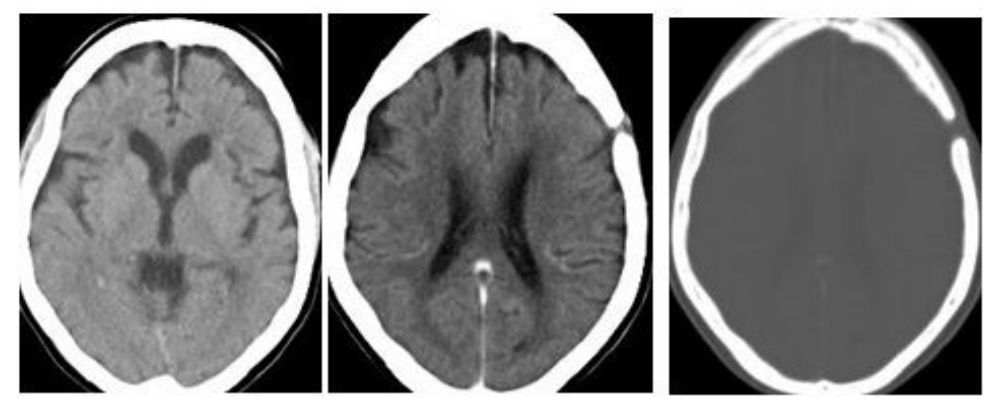

d

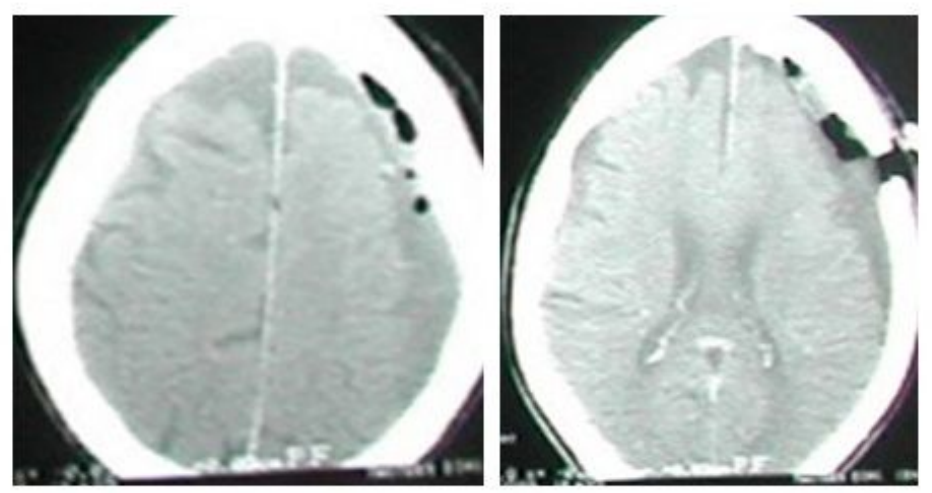

Figure 6

An 82-year-old woman with a (a) left sided mixed density CSDH made brisk clinical and (b) radiological recovery from surgery. Two months later she showed clinical and (c) radiological evidence of recurrence. This was reoperated through the same left sided single frontal burr-hole craniostomy under local anaesthesia. She made another good clinical and (d) radiological recovery. 
a

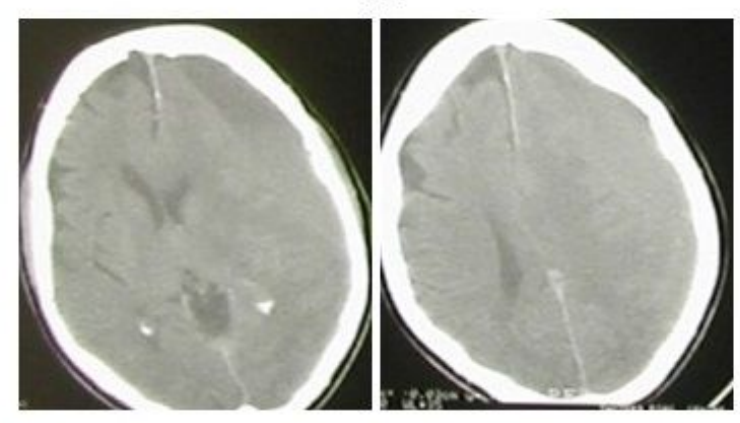

C
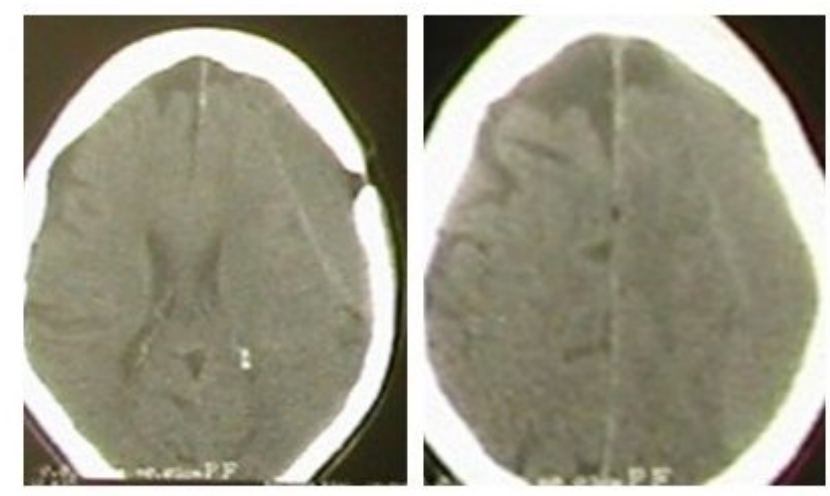

b

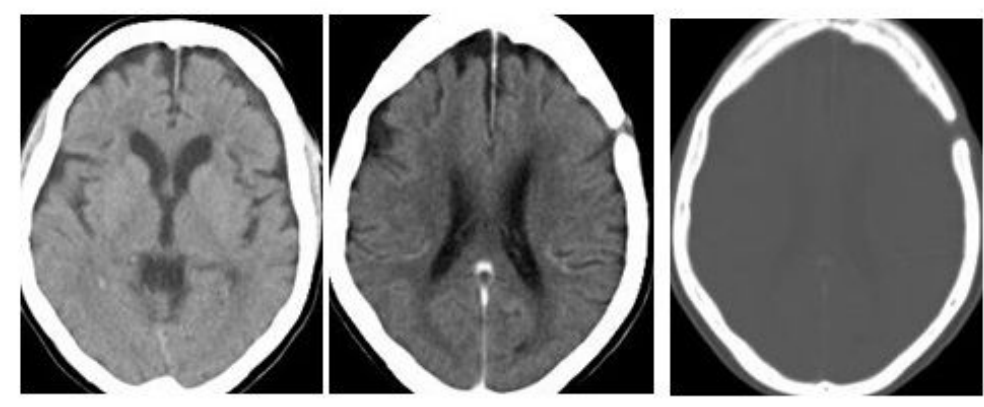

d

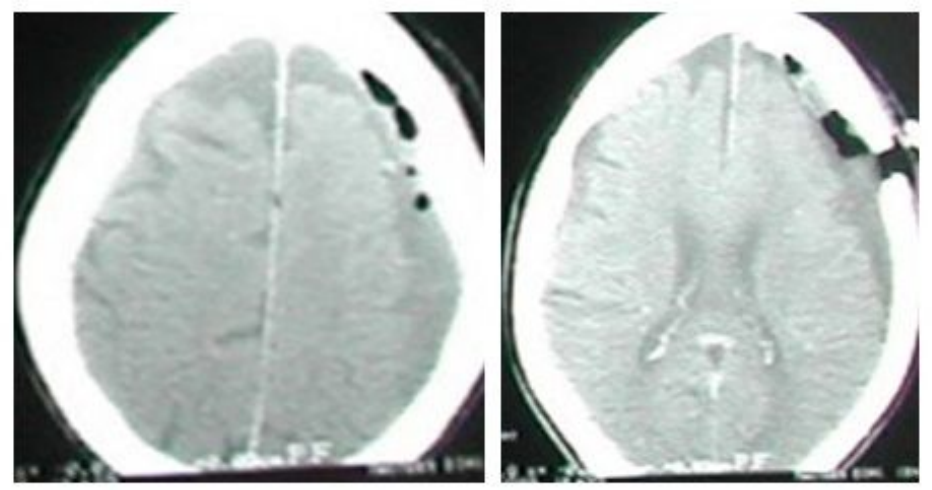

Figure 6

An 82-year-old woman with a (a) left sided mixed density CSDH made brisk clinical and (b) radiological recovery from surgery. Two months later she showed clinical and (c) radiological evidence of recurrence. This was reoperated through the same left sided single frontal burr-hole craniostomy under local anaesthesia. She made another good clinical and (d) radiological recovery. 